\title{
Genus expansion of open free energy in $2 d$ topological gravity
}

\author{
Kazumi Okuyama $^{a}$ and Kazuhiro Sakai ${ }^{b}$ \\ ${ }^{a}$ Department of Physics, Shinshu University, \\ 3-1-1 Asahi, Matsumoto 390-8621, Japan \\ ${ }^{b}$ Institute of Physics, Meiji Gakuin University, \\ 1518 Kamikurata-cho, Totsuka-ku, Yokohama 244-8539, Japan \\ E-mail: kazumi@azusa.shinshu-u.ac.jp, kzhrsakai@gmail.com
}

\begin{abstract}
We study open topological gravity in two dimensions, or, the intersection theory on the moduli space of open Riemann surfaces initiated by Pandharipande, Solomon and Tessler. The open free energy, the generating function for the open intersection numbers, obeys the open KdV equations and Buryak's differential equation and is related by a formal Fourier transformation to the Baker-Akhiezer wave function of the KdV hierarchy. Using these properties we study the genus expansion of the free energy in detail. We construct explicitly the genus zero part of the free energy. We then formulate a method of computing higher genus corrections by solving Buryak's equation and obtain them up to high order. This method is much more efficient than our previous approach based on the saddle point calculation. Along the way we show that the higher genus corrections are polynomials in variables that are expressed in terms of genus zero quantities only, generalizing the constitutive relation of closed topological gravity.
\end{abstract}

KEYwords: 2D Gravity, Integrable Hierarchies, Matrix Models

ArXiv ePrint: 2009.12731 


\section{Contents}

1 Introduction 1

2 Brief review of topological gravity 3

2.1 Witten-Kontsevich closed topological gravity 3

2.2 Pandharipande-Solomon-Tessler open topological gravity 5

2.3 Open free energy and Fourier transform of BA function 6

3 Genus expansion of open free energy $\quad 7$

$\begin{array}{lll}3.1 & \text { Genus expansion of BA function } & 7\end{array}$

3.2 Saddle point calculation and polynomial structure of free energy 8

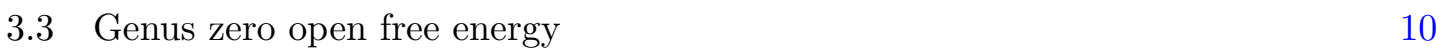

$\begin{array}{lll}3.4 & \text { Recursion relation } & 12\end{array}$

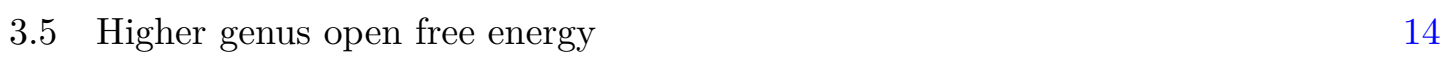

4 Conclusions and outlook $\quad 15$

$\begin{array}{ll}\text { A Derivation of open KdV equations } & 16\end{array}$

$\begin{array}{ll}\text { B Derivation of } z_{*}(s) & 17\end{array}$

\section{Introduction}

Two-dimensional gravity is one of the simplest models of quantum gravity which has been intensively studied for quite some time. In [1-4] it was found that two-dimensional gravity is described by a certain double-scaled random matrix model (see [5] for a review). Mathematically, two-dimensional gravity corresponds to an intersection theory on the moduli space of closed Riemann surfaces, as first conjectured by Witten [6] and proved by Kontsevich [7]. It is known that the free energy of two-dimensional gravity on closed Riemann surfaces satisfies the KdV equations [6-8] and the Virasoro constraints [9, 10]. Recently, it is realized that this story holds for Jackiw-Teitelboim (JT) gravity as well; Saad, Shenker and Stanford [11] showed that JT gravity is described by a doubled-scaled matrix model and it corresponds to a particular background of Witten-Kontsevich topological gravity [12-14].

Recently, Pandharipande, Solomon and Tessler [15] initiated the study of open topological gravity, i.e. the intersection theory on the moduli space of Riemann surfaces with boundary. See also [16-21] for related works. It is conjectured in [15] and proved in [18] that the open free energy $F^{\circ}(s),{ }^{1}$ or the generating function of the open intersection numbers, satisfies the open version of the $\mathrm{KdV}$ equations and the Virasoro constraints. As

\footnotetext{
${ }^{1}$ The variable $s$ is related to the 't Hooft parameter $\lambda$ in our previous papers $[14,22]$ by $\lambda=\sqrt{2} s$.
} 
explained in [13], open topological gravity is physically realized by adding vector degrees of freedom to the matrix model of two-dimensional gravity. After integrating out the vector degrees of freedom, this amounts to the insertion of the determinant operator $\operatorname{det}(\xi-M)$ to the matrix integral, where $\xi$ is a parameter and $M$ is the random matrix. The expectation value of this determinant operator

$$
\psi(\xi)=e^{-\frac{1}{2 g_{\mathrm{s}}} V(\xi)}\langle\operatorname{det}(\xi-M)\rangle
$$

corresponds to the wavefunction of the FZZT brane $[23,24]$. Here $V(\xi)$ is the matrix model potential and $g_{\mathrm{s}}$ is the genus counting parameter (denoted as $u$ in $[15-18]$ ). $\psi(\xi)$ is also identified as the Baker-Akhiezer (BA) function of the KdV hierarchy [25].

It is known that the exponential of the open free energy $e^{F^{\circ}(s)}$ and the BA function $\psi(\xi)$ are related by the formal Fourier transformation $[13,18]^{2}$

$$
e^{F^{\circ}(s)}=\int_{-\infty}^{\infty} d \xi e^{\frac{s \xi}{g_{\mathrm{s}}}} \psi(\xi)
$$

One can compute the small $g_{\mathrm{s}}$ expansion of $F^{\mathrm{o}}(s)$

$$
F^{\mathrm{o}}(s)=\sum_{\tilde{g}=0}^{\infty} g_{\mathrm{s}}^{\tilde{g}-1} F_{\tilde{g}}^{\mathrm{o}}(s)
$$

from the result of the WKB expansion of the BA function by evaluating the integral (1.2) by the saddle point method. For instance, the leading term of the small $g_{\mathrm{s}}$ expansion of $\psi(\xi) \approx e^{-\frac{1}{2 g_{\mathrm{s}}} V_{\mathrm{eff}}(\xi)}$ is given by the so-called effective potential $V_{\text {eff }}(\xi)$. In our previous paper [22], we obtained the explicit form of $V_{\text {eff }}(\xi)$ for arbitrary background couplings $\left\{t_{n}\right\}$. Then the leading term $F_{0}^{o}(s)$ in (1.3) is given by the Legendre transform of $V_{\text {eff }}(\xi)$. One can in principle continue this saddle point computation for the higher order corrections in $g_{\mathrm{s}}$, but the computation becomes very cumbersome as the order of $g_{\mathrm{s}}$ increases.

It turns out that the small $g_{\mathrm{s}}$ expansion of $F^{\circ}(s)$ can be computed systematically by recursively solving Buryak's equation [17], which is understood as the Fourier transform of the Schrödinger equation of the KdV hierarchy. This is based on the fact that $F_{\tilde{g} \geq 2}^{o}(s)$ in (1.3) is written as a polynomial in variables which are expressed in terms of genus zero quantities only. This is similar to the situation in original Witten-Kontsevich topological gravity, where the genus- $g(\geq 2)$ closed free energy $F_{g}^{c}$ is written as a polynomial in a certain basis $[26,27]$.

This paper is organized as follows. In section 2 we briefly review closed and open topological gravities. We also explain how the open KdV equations and Buryak's equation are derived from the KdV hierarchy. In section 3 we study the genus expansion of the open free energy. We first compute it from the genus expansion of the BA function by the saddle point calculation. We next derive an explicit expression of the genus zero open free energy. We then formulate a method of computing the genus expansion by solving Buryak's equation. We conclude in section 4 with discussions on the future directions. Some details of the calculations are relegated to the appendices A and B.

\footnotetext{
${ }^{2}$ The inverse transformation of (1.2) is considered in [18]. Our (1.2) is equivalent to eq. (4.76) in [13].
} 


\section{Brief review of topological gravity}

In this section we will briefly review the basics and known results about closed and open topological gravities. We will also explain how the open KdV equations and Buryak's equation, which will be the main tools of our study of the open free energy, are derived from the KdV hierarchy.

\subsection{Witten-Kontsevich closed topological gravity}

In Witten-Kontsevich topological gravity [6, 7] (see also [13]) observables are made up of the intersection numbers

$$
\left\langle\tau_{d_{1}} \cdots \tau_{d_{n}}\right\rangle_{g, n}=\int_{\overline{\mathcal{M}}_{g, n}} \psi_{1}^{d_{1}} \cdots \psi_{n}^{d_{n}}, \quad d_{1}, \ldots, d_{n} \in \mathbb{Z}_{\geq 0} .
$$

They are defined on a closed Riemann surface $\Sigma$ of genus $g$ with $n$ marked points $p_{1}, \ldots, p_{n}$. We let $\mathcal{M}_{g, n}$ denote the moduli space of $\Sigma$ and $\overline{\mathcal{M}}_{g, n}$ the Deligne-Mumford compactification of $\mathcal{M}_{g, n}$. Here $\tau_{d_{i}}=\psi_{i}^{d_{i}}$ and $\psi_{i}$ is the first Chern class of the complex line bundle whose fiber is the cotangent space to $p_{i}$. The intersection numbers (2.1) obey the selection rule

$$
\left\langle\tau_{d_{1}} \cdots \tau_{d_{n}}\right\rangle_{g, n}=0 \quad \text { unless } \quad d_{1}+\cdots+d_{n}=3 g-3+n .
$$

The generating function for the above intersection numbers is defined as

$$
F^{\mathrm{c}}\left(\left\{t_{k}\right\}, g_{\mathrm{s}}\right):=\sum_{g=0}^{\infty} g_{\mathrm{s}}^{2 g-2} F_{g}^{\mathrm{c}}\left(\left\{t_{k}\right\}\right), \quad F_{g}^{\mathrm{c}}\left(\left\{t_{k}\right\}\right):=\left\langle e^{\sum_{d=0}^{\infty} t_{d} \tau_{d}}\right\rangle_{g} .
$$

We will call $F^{\mathrm{c}}$ the closed free energy.

It was conjectured [6] and proved [7] that $e^{F^{\mathrm{c}}}$ is a tau function for the $\mathrm{KdV}$ hierarchy. This means that

$$
u:=g_{\mathrm{s}}^{2} \partial_{0}^{2} F^{\mathrm{c}}
$$

satisfies the $\mathrm{KdV}$ equations

$$
\partial_{k} u=\partial_{0} \mathcal{R}_{k+1}
$$

where $\mathcal{R}_{k}$ are the Gelfand-Dikii differential polynomials of $u$

$$
\mathcal{R}_{0}=1, \quad \mathcal{R}_{1}=u, \quad \mathcal{R}_{2}=\frac{u^{2}}{2}+\frac{g_{\mathrm{s}}^{2} \partial_{0}^{2} u}{12}, \cdots .
$$

Here we have introduced the notation

$$
\partial_{k}:=\frac{\partial}{\partial t_{k}} .
$$

For $k=1,(2.5)$ gives the traditional KdV equation

$$
\partial_{1} u=u \partial_{0} u+\frac{g_{\mathrm{s}}^{2}}{12} \partial_{0}^{3} u
$$


Integrating (2.5) once in $t_{0}$ we have

$$
g_{\mathrm{s}}^{2} \partial_{k} \partial_{0} F^{\mathrm{c}}=\mathcal{R}_{k+1} .
$$

It is well known (see e.g. [28]) that the KdV equations (2.5) are obtained as the compatibility condition of the Schrödinger equation

$$
Q \psi=\xi \psi
$$

and the KdV flow equations

$$
\partial_{k} \psi=M_{k} \psi
$$

where

$$
Q:=\frac{g_{\mathrm{s}}^{2}}{2} \partial_{0}^{2}+u, \quad M_{k}:=\frac{(2 Q)_{+}^{k+1 / 2}}{(2 k+1) ! ! g_{\mathrm{s}}}
$$

Here we have decomposed $(2 Q)^{k+1 / 2}=(2 Q)_{+}^{k+1 / 2}+(2 Q)_{-}^{k+1 / 2}$ and the subscript + means that $(2 Q)_{+}^{k+1 / 2}$ contains only non-negative powers of $\partial_{0}$. Indeed, $(2.5)$ is recovered by using the relation

$$
\left[M_{k}, Q\right]=\partial_{0} \mathcal{R}_{k+1}
$$

The wave function $\psi$ that satisfies (2.10) and (2.11) is known as the Baker-Akhiezer function.

Another important constraint that the closed free energy $F^{\mathrm{c}}$ obeys is the string equation [8]. For Witten-Kontsevich gravity it is written as

$$
u-\sum_{k=0}^{\infty} t_{k} \mathcal{R}_{k}=0
$$

The genus zero part of this string equation is written as

$$
u_{0}-I_{0}\left(u_{0},\left\{t_{k}\right\}\right)=0,
$$

where $u_{0}$ is the genus-zero part of $u$

$$
u_{0}:=\partial_{0}^{2} F_{0}^{\mathrm{c}}
$$

and we have introduced the Itzykson-Zuber variables [26]

$$
I_{n}\left(v,\left\{t_{k}\right\}\right)=\sum_{\ell=0}^{\infty} t_{n+\ell} \frac{v^{\ell}}{\ell !} \quad(n \geq 0) .
$$

Throughout this paper $I_{n}$ without specifying its arguments should always be understood as

$$
I_{n}=I_{n}\left(u_{0},\left\{t_{k}\right\}\right)
$$


It is also convenient to introduce the variable

$$
t:=\left(\partial_{0} u_{0}\right)^{-1}=1-I_{1}
$$

It was conjectured [26] and proved $[27,29]$ that $F_{g}\left(\left\{t_{k}\right\}\right)(g \geq 2)$ are polynomials in $I_{n \geq 2}$ and $t^{-1}$. This fact significantly helps us to compute higher genus free energy $F_{g}^{\mathrm{c}}$.

An efficient way to compute $F_{g}^{\mathrm{c}}$ is as follows. (See [14] for a more detailed explanation.) Let us expand $u$ as

$$
u=\sum_{g=0}^{\infty} g_{\mathrm{s}}^{2 g} u_{g}, \quad u_{g}=\partial_{0}^{2} F_{g}^{\mathrm{c}}
$$

$u_{g}$ can be computed by recursively solving the KdV equation (2.8). To do this, let us regard $t_{k \geq 2}$ as parameters and consider the change of variables from $\left(t_{0}, t_{1}\right)$ to $\left(u_{0}, t\right)$. The differentials $\partial_{0,1}$ are then written in the new variables as ${ }^{3}$

$$
\partial_{0}=\frac{1}{t}\left(\partial_{u_{0}}-I_{2} \partial_{t}\right), \quad \partial_{1}=u_{0} \partial_{0}-\partial_{t}
$$

By expanding both sides of the equation in $g_{\mathrm{s}}(2.8)$ is written as the recursion relation

$$
-\frac{1}{t} \partial_{t}\left(t u_{g}\right)=\sum_{h=1}^{g-1} u_{g-h} \partial_{0} u_{h}+\frac{1}{12} \partial_{0}^{3} u_{g-1} \quad(g \geq 1) .
$$

This is easily solved with the help of (2.21). First few of $u_{g}$ are

$$
\begin{aligned}
& u_{1}=\frac{I_{2}^{2}}{12 t^{4}}+\frac{I_{3}}{24 t^{3}}, \\
& u_{2}=\frac{49 I_{2}^{5}}{288 t^{9}}+\frac{11 I_{2}^{3} I_{3}}{36 t^{8}}+\frac{84 I_{2}^{2} I_{4}+109 I_{2} I_{3}^{2}}{1152 t^{7}}+\frac{32 I_{2} I_{5}+51 I_{3} I_{4}}{2880 t^{6}}+\frac{I_{6}}{1152 t^{5}} .
\end{aligned}
$$

As explained in [14] one can easily integrate $u_{g}$ twice in $t_{0}$ and obtain the well-known results [26]

$$
\begin{aligned}
& F_{1}^{\mathrm{c}}=-\frac{1}{24} \log t \\
& F_{2}^{\mathrm{c}}=\frac{I_{4}}{1152 t^{3}}+\frac{29 I_{2} I_{3}}{5760 t^{4}}+\frac{7 I_{2}^{3}}{1440 t^{5}} .
\end{aligned}
$$

\subsection{Pandharipande-Solomon-Tessler open topological gravity}

Pandharipande, Solomon and Tessler proposed an open analog of Witten-Kontsevich topological gravity [15]. They introduced the open intersection numbers

$$
\left\langle\tau_{d_{1}} \cdots \tau_{d_{n}} \sigma^{k}\right\rangle_{\tilde{g}, n}^{\mathrm{o}}=2^{-\frac{\tilde{g}+k-1}{2}} \int_{\overline{\mathcal{M}}_{\tilde{g}, k, n}} e(E, s), \quad d_{1}, \ldots, d_{n} \in \mathbb{Z}_{\geq 0}
$$

The new insertion $\sigma$ corresponds to the addition of a boundary marking and the power $k$ of $\sigma$ specifies the number of boundary markings. $e(E, s)$ is the relative Euler class [15],

\footnotetext{
${ }^{3}$ This change of variables was originally introduced by Zograf (see e.g. [30]).
} 
which is thought of as an open analog of the Euler class $e(E)=\psi_{1}^{d_{1}} \cdots \psi_{n}^{d_{n}}$ used in (2.1). $\overline{\mathcal{M}}_{\tilde{g}, k, n}$ denotes a suitable compactification of the moduli space $\mathcal{M}_{\tilde{g}, k, n}$ of Riemann surfaces with boundary of doubled genus $\tilde{g}$ with graded spin structures, ${ }^{4} n$ interior and $k$ boundary marked points. The open intersection numbers (2.25) obey the selection rule

$$
\left\langle\tau_{d_{1}} \cdots \tau_{d_{n}} \sigma^{k}\right\rangle_{\tilde{g}, n}^{\mathrm{O}}=0 \quad \text { unless } \quad 2 \sum_{j=1}^{n} d_{j}=3 \tilde{g}-3+k+2 n .
$$

The generating function for the open intersection numbers is defined as

$$
F^{\mathrm{o}}\left(s,\left\{t_{k}\right\}, g_{\mathrm{s}}\right):=\sum_{\tilde{g}=0}^{\infty} g_{\mathrm{s}}^{\tilde{g}-1} F_{\tilde{g}}^{\mathrm{o}}\left(s,\left\{t_{k}\right\}\right), \quad F_{\tilde{g}}^{\mathrm{o}}\left(s,\left\{t_{k}\right\}\right):=\left\langle e^{s \sigma+\sum_{d=0}^{\infty} t_{d} \tau_{d}}\right\rangle_{\tilde{g}}^{\mathrm{o}} .
$$

We will call $F^{\mathrm{o}}$ the open free energy.

It was conjectured [15] and then proved [18] that $F^{\mathrm{o}}$ satisfies the open $\mathrm{KdV}$ equations

$$
\frac{2 n+1}{2} \partial_{n} F^{\mathrm{o}}=g_{\mathrm{s}} \partial_{s} F^{\mathrm{o}} \partial_{n-1} F^{\mathrm{o}}+g_{\mathrm{s}} \partial_{s} \partial_{n-1} F^{\mathrm{o}}+\frac{g_{\mathrm{s}}^{2}}{2} \partial_{0} F^{\mathrm{o}} \partial_{0} \partial_{n-1} F^{\mathrm{c}}-\frac{g_{\mathrm{s}}^{2}}{4} \partial_{0}^{2} \partial_{n-1} F^{\mathrm{c}} \quad(n \geq 1) .
$$

In fact it is known [17] that $F^{\circ}$ is fully determined by the above system of equations with the initial condition

$$
\left.F^{\mathrm{o}}\right|_{t_{k \geq 1}=0}=\frac{1}{g_{\mathrm{s}}}\left(\frac{s^{3}}{6}+t_{0} s\right)
$$

given the closed free energy $F^{c}$. Buryak proved that $F^{\circ}$ further satisfies another differential equation [17]

$$
\partial_{s} F^{\mathrm{o}}=g_{\mathrm{s}}\left[\frac{1}{2}\left(\partial_{0} F^{\mathrm{o}}\right)^{2}+\frac{1}{2} \partial_{0}^{2} F^{\mathrm{o}}+\partial_{0}^{2} F^{\mathrm{c}}\right] .
$$

These equations play a crucial role in the study of $F^{\circ}$ in this paper.

In [16] Buryak constructed an explicit expression for $e^{F^{\circ}}$ in terms of $F^{\mathrm{c}}$. In this sense an explicit form of $F^{\circ}$ is known. For many purposes, however, it is still useful to express $F^{\circ}$ in the form of genus expansion (2.27) and construct an explicit, closed expression of $F_{\tilde{g}}^{o}\left(s,\left\{t_{k}\right\}\right)$ for fixed $\tilde{g}$. This is our primary goal in this paper.

\subsection{Open free energy and Fourier transform of BA function}

It is known $[13,18]$ that the exponential of the open free energy $\exp F^{\circ}$ is related to the BA function $\psi$ by the formal Fourier transformation (1.2). Using this relation one can show [18] that the open KdV equations (2.28) and Buryak's equation (2.30) are in fact derived from the KdV flow equations (2.11) and the Schrödinger equation (2.10) respectively. In what follows we will present a derivation in a manner slightly different from [18].

Let $\widehat{\psi}$ denote the formal Fourier transform of the BA function $\psi$

$$
\widehat{\psi}(s):=\int_{-\infty}^{\infty} d \xi e^{\frac{s \xi}{g_{\mathrm{s}}}} \psi(\xi) .
$$

\footnotetext{
${ }^{4}$ For the notion of graded spin structures, see e.g. [19].
} 
In terms of $\widehat{\psi}$ the Schrödinger equation (2.10) is written as

$$
Q \widehat{\psi}=g_{\mathrm{s}} \partial_{s} \widehat{\psi}
$$

On the other hand, (1.2) is written as

$$
\widehat{\psi}=e^{F^{\mathrm{o}}} \text {. }
$$

Substituting (2.33) into (2.32) one obtains

$$
\frac{g_{\mathrm{s}}^{2}}{2} \partial_{0}^{2} e^{F^{\circ}}+u e^{F^{\circ}}=g_{\mathrm{s}} \partial_{s} e^{F^{\circ}} .
$$

Rewriting $u$ by (2.4) we immediately see that this is equivalent to (2.30). We have thus seen that Buryak's equation (2.30) is nothing but the formal Fourier transform of the Schrödinger equation (2.10).

Similarly, let us consider the Fourier transform of the KdV flow equations (2.11). It is clear that the same equations hold for $\widehat{\psi}$ as well

$$
\begin{aligned}
\partial_{n} \widehat{\psi} & =M_{n} \widehat{\psi} \\
& =\frac{(2 Q)_{+}^{n+\frac{1}{2}}}{(2 n+1) ! ! g_{\mathrm{s}}} \widehat{\psi}
\end{aligned}
$$

It is shown [16] that these equations are satisfied by $\widehat{\psi}$ in (2.33) with $F^{\circ}$ obeying the open $\mathrm{KdV}$ equations (2.11). Conversely, we can directly derive the open KdV equations (2.11) from (2.35) using (2.33). Since the derivation is rather technical, we relegate it to appendix A. We stress that the open $\mathrm{KdV}$ equations are equivalent to the $\mathrm{KdV}$ flow equations (2.11) under the identification (2.33).

\section{Genus expansion of open free energy}

In this section we will study the genus expansion of the open free energy. We will first compute it from the genus expansion of the BA function by the saddle point calculation. We will next derive a fully explicit expression of the genus zero open free energy. Finally, we will formulate a method of computing the genus expansion by solving Buryak's equation, which turns out to be much more efficient than the saddle point calculation.

\subsection{Genus expansion of BA function}

We saw in [22] that the BA function $\psi$ admits the following expansion

$$
\psi=e^{A}, \quad A=\sum_{\tilde{g}=0}^{\infty} g_{\mathrm{s}}^{\tilde{g}-1} A_{\tilde{g}}
$$


First few of $A_{\tilde{g}} \operatorname{are}^{5}$

$$
\begin{aligned}
& A_{0}=-\frac{t z^{3}}{3}+\sum_{n=1}^{\infty} \frac{I_{n+1}}{(2 n+3) ! !} z^{2 n+3} \\
& A_{1}=-\frac{1}{2} \log z+\text { const. } \\
& A_{2}=-\frac{5}{24 t z^{3}}-\frac{I_{2}}{24 t^{2} z}, \\
& A_{3}=\frac{5}{16 t^{2} z^{6}}+\frac{1}{t^{3}}\left(\frac{I_{2}}{8 z^{4}}+\frac{I_{3}}{48 z^{2}}\right)+\frac{I_{2}^{2}}{24 t^{4} z^{2}}
\end{aligned}
$$

where we have introduced ${ }^{6}$

$$
z:=\sqrt{2\left(\xi-u_{0}\right)}
$$

$A_{\tilde{g}}$ can be computed up to any order by solving the recursion relation for $v_{\tilde{g}}:=\partial_{0} A_{\tilde{g}}$

$$
\begin{aligned}
& v_{\tilde{g}}=-\frac{1}{2 v_{0}}\left(\partial_{0} v_{\tilde{g}-1}+\sum_{k=1}^{\tilde{g}-1} v_{k} v_{\tilde{g}-k}+\left\{\begin{array}{ll}
2 u_{\frac{\tilde{g}}{2}} & (\tilde{g} \text { even }) \\
0 & (\tilde{g} \text { odd })
\end{array}\right), \quad(n \geq 2),\right. \\
& v_{0}=z, \quad v_{1}=\frac{1}{2 t z^{2}}
\end{aligned}
$$

In [22] we performed this computation with special values of $t_{k}$ corresponding to the case of JT gravity, but as advertised in [31] it can be generalized without any effort to the case of general values of $t_{k}$, as we have seen above.

\subsection{Saddle point calculation and polynomial structure of free energy}

The Fourier transformation (1.2) enables us to calculate the genus expansion (1.3) of $F^{\mathrm{o}}$ from that of $A=\log \psi$ just obtained above. (1.2) is written as

$$
\begin{aligned}
e^{F^{\mathrm{o}}} & =\int_{-\infty}^{\infty} d \xi e^{\frac{s \xi}{g_{\mathrm{s}}}+A} \\
& =\int_{-\infty}^{\infty} d \xi e^{\left[s \xi+A_{0}(\xi)\right] g_{\mathrm{s}}^{-1}+A_{1}(\xi)+A_{2}(\xi) g_{\mathrm{s}}+\mathcal{O}\left(g_{\mathrm{s}}^{2}\right)} .
\end{aligned}
$$

As in $[14,22]$ one can calculate $F_{\tilde{g}}^{o}$ by the saddle point method.

The saddle point $\xi_{*}$ is given by the condition

$$
\left.\partial_{\xi}\left[s \xi+A_{0}(\xi)\right]\right|_{\xi=\xi_{*}}=0
$$

\footnotetext{
${ }^{5}$ In [22] the constant part of $A_{1}$ is fixed so that it fits well with the convention of closed topological gravity. In this paper we will use this degree of freedom later for compensating the difference of the normalizations of $e^{F^{\circ}}$ and $\psi$, so that we can avoid putting an inessential normalization factor in (1.2).

${ }^{6} A_{\tilde{g}}, v_{\tilde{g}}$ and $z$ in this paper are related to those in our previous paper [22] by $A_{\tilde{g}}^{\text {here }}=(\sqrt{2})^{1-\tilde{g}} A_{\tilde{g}}^{\text {there }}$, $v_{\tilde{g}}^{\text {here }}=(\sqrt{2})^{1-\tilde{g}} v_{\tilde{g}}^{\text {there }}, z^{\text {here }}=\sqrt{2} z^{\text {there }}$.
} 
This is equivalent to

$$
\begin{aligned}
s & =-\left.\partial_{\xi} A_{0}\right|_{\xi=\xi_{*}} \\
& =t z_{*}-\sum_{n=1}^{\infty} \frac{I_{n+1}}{(2 n+1) ! !} z_{*}^{2 n+1},
\end{aligned}
$$

where

$$
z_{*}:=\sqrt{2\left(\xi_{*}-u_{0}\right)} \quad \Leftrightarrow \quad \xi_{*}=\frac{z_{*}^{2}}{2}+u_{0}
$$

By using the Lagrange inversion theorem this is inverted as (see appendix B)

$$
z_{*}=\sum_{\substack{j_{a} \geq 0 \\ \sum_{a} j_{a}=k \\ \sum_{a} a j_{a}=n}} \frac{(2 n+k) !}{(2 n+1) !} \frac{s^{2 n+1}}{t^{2 n+k+1}} \prod_{a=1}^{\infty} \frac{I_{a+1}^{j_{a}}}{j_{a} !(2 a+1) ! ! j_{a}} .
$$

As in [14] let us introduce a new variable $\phi$ as

$$
\xi=\xi_{*}+\sqrt{g_{\mathrm{s}}} \phi
$$

The integral (3.5) is then written as

$$
e^{F^{\circ}}=e^{\left[s \xi_{*}+A_{0}\left(\xi_{*}\right)\right] g_{\mathrm{s}}^{-1}+A_{1}\left(\xi_{*}\right)} \int_{-\infty}^{\infty} \sqrt{g_{\mathrm{s}}} d \phi e^{\frac{1}{2} \partial_{\xi_{*}}^{2} A_{0}\left(\xi_{*}\right) \phi^{2}+\mathcal{O}\left(g_{\mathrm{s}}^{1 / 2}\right)} .
$$

By expanding the integrand in $g_{\mathrm{s}}$, the integral in $\phi$ can be performed order by order as a Gaussian integral. In fact, we did essentially the same calculation in [14] up to the order of $g_{\mathrm{s}}^{1}$. We thus immediately obtain

$$
\begin{aligned}
F_{0}^{\mathrm{o}} & =s \xi_{*}+A_{0}\left(\xi_{*}\right)=s \xi_{*}-\int_{u_{0}}^{\xi_{*}} d \xi_{*}^{\prime} s\left(\xi_{*}^{\prime}\right) \\
& =\int_{0}^{s} d s^{\prime} \xi_{*}\left(s^{\prime}\right) \\
F_{1}^{\mathrm{o}} & =A_{1}\left(\xi_{*}\right)+\frac{1}{2} \log \frac{2 \pi g_{\mathrm{s}}}{-\partial_{\xi_{*}}^{2} A_{0}\left(\xi_{*}\right)} \\
& =\frac{1}{2} \log \frac{\partial_{s} \xi_{*}}{z_{*}}=\frac{1}{2} \log \partial_{s} z_{*}, \\
F_{2}^{\mathrm{o}} & =A_{2}\left(\xi_{*}\right)+\frac{1}{2}\left(\frac{A_{0 *}^{(3)}}{3 !}\right)^{2}\left\langle\phi^{6}\right\rangle+\left(\frac{A_{0 *}^{(4)}}{4 !}+\frac{A_{0 *}^{(3)} A_{1 *}^{(1)}}{3 !}\right)\left\langle\phi^{4}\right\rangle+\frac{\left(A_{1 *}^{(1)}\right)^{2}+A_{1 *}^{(2)}}{2}\left\langle\phi^{2}\right\rangle \\
& =-\frac{5}{24 t z_{*}^{3}}-\frac{I_{2}}{24 t^{2} z_{*}}+\frac{\xi_{*}^{(3)}}{8\left(\xi_{*}^{(1)}\right)^{2}}-\frac{\left(\xi_{*}^{(2)}\right)^{2}}{6\left(\xi_{*}^{(1)}\right)^{3}}-\frac{\xi_{*}^{(2)}}{4 z_{*}^{2} \xi_{*}^{(1)}}+\frac{5 \xi_{*}^{(1)}}{8 z_{*}^{4}},
\end{aligned}
$$

where we have introduced the notation

$$
A_{\tilde{g} *}^{(n)}:=\left.\partial_{\xi}^{n} A_{\tilde{g}}\right|_{\xi=\xi_{*}}, \quad \xi_{*}^{(n)}:=\partial_{s}^{n} \xi_{*} .
$$


In the last equality in (3.12) we have used

$$
\begin{aligned}
A_{0 *}^{(n)} & =-\left(\frac{1}{\xi_{*}^{(1)}} \frac{\partial}{\partial s}\right)^{n-1} s \quad(n \geq 1), \\
\left\langle\phi^{2 m}\right\rangle & =\frac{\int_{-\infty}^{\infty} d \phi e^{\frac{1}{2} A_{0 *}^{(2)} \phi^{2}} \phi^{2 m}}{\int_{-\infty}^{\infty} d \phi e^{\frac{1}{2} A_{0 *}^{(2)} \phi^{2}}}=\frac{(2 m-1) ! !}{\left(-A_{0 *}^{(2)}\right)^{m}}=(2 m-1) ! !\left(\xi_{*}^{(1)}\right)^{m} \quad(m \geq 1) .
\end{aligned}
$$

We have fixed the constant part of $A_{1}$ in (3.2) in such a way that the initial condition (2.29) is satisfied. Using this method one can in principle calculate $F_{\tilde{g}}^{o}$ up to any order. However, this calculation gets quickly involved as $\tilde{g}$ increases. We will propose an alternative, much more efficient method of computing $F_{\tilde{g}}^{o}$ in the following subsections.

An advantage of the above calculation is that we can prove the polynomial structure of the higher genus free energies $F_{\tilde{g} \geq 2}^{o}$. The expansion (3.11) implies that $F_{\tilde{g} \geq 2}^{o}$ are polynomials in $A_{0 *}^{(n \geq 3)}, A_{1 *}^{(n \geq 1)}, A_{\tilde{g} \geq 2 *}^{(n \geq 0)}$ and $\left\langle\phi^{2 m}\right\rangle(m \geq 1)$. On the other hand, by using the polynomial structure of the closed free energy reviewed in section 2.1, it is easy to show that $A_{\tilde{g} \geq 2}$ are polynomials in $t^{-1}, I_{k \geq 2}$ and $z^{-1}$. Combining these two lemmas we arrive at the conclusion that $F_{\tilde{g} \geq 2}^{\mathrm{O}}$ are polynomials in the variables $t^{-1}, I_{k \geq 2}, z_{*}^{-1},\left(\xi_{*}^{(1)}\right)^{-1}$ and $\xi_{*}^{(n \geq 1)}$.

It is well known that closed topological gravity exhibits the constitutive relation [32], i.e. higher genus quantities are expressed in terms of genus zero quantities only. In the case of Witten-Kontsevich gravity $F_{1}^{c}$ is given as in (2.24) and $F_{g \geq 2}^{c}$ are expressed as polynomials in the variables $t^{-1}$ and $I_{k \geq 2}$, as we saw in section 2.1. These variables are expressed explicitly in terms of genus zero quantities $\partial_{0}^{n} u_{0}(n \geq 1)$ [33]. Since $z_{*}$ and $\xi_{*}^{(n)}=\partial_{s}^{n+1} F_{0}^{\mathrm{o}}$ are also genus zero quantities, ${ }^{7}$ the form of $F_{1}^{\mathrm{o}}$ in $(3.12)$ and the above polynomial structure of $F_{\tilde{g} \geq 2}^{\mathrm{o}}$ ensure that Pandharipande-Solomon-Tessler open topological gravity exhibits a generalized constitutive relation.

\subsection{Genus zero open free energy}

In the last subsection we have obtained an explicit expression of $F_{0}^{o}$ : by plugging (3.8) into the second line of (3.12) we have

$$
F_{0}^{\mathrm{o}}=u_{0} s+\frac{1}{2} \int_{0}^{s} d s^{\prime} z_{*}\left(s^{\prime}\right)^{2}
$$

with $z_{*}(s)$ given in (3.9). As we will see below, we can write down a more direct expression for $F_{0}^{\mathrm{o}}$ by using the relations among $F_{0}^{\mathrm{o}}, \xi_{*}$ and $z_{*}$ which follow from the system of equations (2.30) and (2.28).

Buryak's equation (2.30) at the order of $g_{\mathrm{s}}^{-1}$ reads

$$
\partial_{s} F_{0}^{\mathrm{o}}=\frac{1}{2}\left(\partial_{0} F_{0}^{\mathrm{o}}\right)^{2}+u_{0}
$$

Note also that the second line of (3.12) gives

$$
\partial_{s} F_{0}^{\mathrm{o}}=\xi_{*} .
$$

\footnotetext{
${ }^{7}$ See (3.18). It is also possible to express $\xi_{*}^{(n)}$ in terms of $t_{0}$-derivatives only. This is done by repeatedly using (3.33).
} 
Comparing these with $(3.8)$ one finds ${ }^{8}$

$$
\partial_{0} F_{0}^{\mathrm{o}}=z_{*}
$$

On the other hand, the open $\mathrm{KdV}$ equation (2.28) for $n=1$ at the order of $g_{\mathrm{s}}^{-1}$ reads

$$
\frac{3}{2} \partial_{1} F_{0}^{\mathrm{o}}=\partial_{s} F_{0}^{\mathrm{o}} \partial_{0} F_{0}^{\mathrm{o}}+\frac{1}{2} \partial_{0} F_{0}^{\mathrm{o}} \partial_{0}^{2} F_{0}^{\mathrm{c}} .
$$

By using (2.21), (3.16), (3.18) and (2.16) this becomes

$$
-\partial_{t} F_{0}^{\mathrm{o}}=\frac{z_{*}^{3}}{3}
$$

Applying $\partial_{s}$ to both sides of the equation and using again (3.16) and (3.18) one obtains

$$
-\partial_{t}\left(\frac{z_{*}^{2}}{2}+u_{0}\right)=z_{*}^{2} \partial_{s} z_{*}
$$

which gives

$$
\begin{aligned}
-\partial_{t} z_{*} & =z_{*} \partial_{s} z_{*} \\
& =\partial_{s} \xi_{*} \\
& =\partial_{s}^{2} F_{0}^{\mathrm{o}} .
\end{aligned}
$$

Hence, differentiating (3.9) once in $t$ and then integrating it twice in $s$, one obtains

$$
F_{0}^{\mathrm{o}}=u_{0} s+\sum_{\substack{j_{a} \geq 0 \\ \sum_{a} j_{a}=k}} \frac{(2 n+k+1) !}{(2 n+3) !} \frac{s^{2 n+3}}{t^{2 n+k+2} \prod_{a=1} a j_{a}=n} \prod^{\infty} \frac{I_{a+1}^{j_{a}}}{j_{a} !(2 a+1) ! ! j_{a}} .
$$

The integration constants have been fixed accordingly so that (3.23) matches with (3.15). We verified by series expansion in $s$ that (3.23) and (3.15) are indeed in perfect agreement. Note that when $t_{k \geq 1}=0$, we have $t_{0}=u_{0}, t=1, I_{k \geq 2}=0$ and thus the above $F_{0}^{o}$ becomes

$$
F_{0}^{\mathrm{o}}=t_{0} s+\frac{s^{3}}{6}
$$

This is consistent with the initial condition (2.29).

One can recast (3.23) into another simple form. Using

$$
\frac{1}{t^{2 n+k+2}}=\left(1-I_{1}\right)^{-2 n-k-2}=\sum_{j_{0}=0}^{\infty} \frac{\left(2 n+k+j_{0}+1\right) !}{(2 n+k+1) !} \frac{I_{1}^{j_{0}}}{j_{0} !}
$$

\footnotetext{
${ }^{8}$ Note that $z_{*}$ is the uniformization coordinate on the spectral curve. In the context of minimal string theory, it is known that $z_{*}$ is given by the $t_{0}$-derivative of the disk amplitude $F_{0}^{\mathrm{o}}$ [25].
} 
and rewriting $a+1, j_{\ell-1}, k+j_{0}$ as $a, j_{\ell}, k$ respectively one obtains

$$
F_{0}^{\mathrm{O}}=u_{0} s+\sum_{\substack{j_{a} \geq 0 \\ \sum_{a} j_{a}=k \\ \sum_{a}(a-1) j_{a}=n}} \frac{(2 n+k+1) !}{(2 n+3) !} s^{2 n+3} \prod_{a=1}^{\infty} \frac{I_{a}^{j_{a}}}{j_{a} !(2 a-1) ! ! j_{a}} .
$$

This expression can be directly compared with the result of [15]. By observing

$$
u_{0}=t_{0}+t_{0} \cdot \mathcal{O}\left(t_{*}\right), \quad I_{n}=t_{n}+t_{0} \cdot \mathcal{O}\left(t_{*}\right),
$$

and recalling the definition (2.27) with the selection rule (2.26), it is easy to see that (3.26) indeed reproduces the Theorem 1.4 of [15]

$$
\left\langle\tau_{d_{1}} \cdots \tau_{d_{\ell}} \sigma \sum_{i=1}^{\ell} 2\left(d_{i}-1\right)+3\right\rangle_{0, \ell}^{o}=\frac{\left(\sum_{i=1}^{\ell} 2 d_{i}-\ell+1\right) !}{\prod_{i=1}^{\ell}\left(2 d_{i}-1\right) ! !}, \quad d_{1}, \ldots, d_{\ell} \geq 1 .
$$

Note that (3.26) contains not only this formula but also all the information about open intersection numbers involving the operator $\tau_{0}$.

\subsection{Recursion relation}

By substituting the genus expansions (1.3) and (2.20) Buryak's equation (2.30) at the order of $g_{\mathrm{s}}^{\tilde{g}-1}(\tilde{g} \geq 1)$ is written as

$$
\mathcal{D} F_{\tilde{g}}^{\mathrm{o}}=\frac{1}{2} \sum_{k=1}^{\tilde{g}-1} \partial_{0} F_{\tilde{g}-k}^{\mathrm{o}} \partial_{0} F_{k}^{\mathrm{o}}+\frac{1}{2} \partial_{0}^{2} F_{\tilde{g}-1}^{\mathrm{o}}+\left\{\begin{array}{ll}
u_{\frac{\tilde{g}}{2}} & (\tilde{g} \text { even }) \\
0 & (\tilde{g} \text { odd })
\end{array},\right.
$$

where we have introduced the differential operator

$$
\mathcal{D}:=\partial_{s}-z_{*} \partial_{0} .
$$

(3.29) can be viewed as a recursion relation: one can recursively compute $F_{\tilde{g}}^{\mathrm{o}}$ if one is able to perform the integration $\mathcal{D}^{-1}$ on the l.h.s. of (3.29). This is indeed feasible, as we will see below.

To do this, let us first study the operator $\mathcal{D}$, which has in fact several interesting properties. For instance, one can show that

$$
\begin{aligned}
\mathcal{D} z_{*} & =\frac{1}{t}, \\
\mathcal{D} \xi_{*} & =0 \\
\mathcal{D} \xi_{*}^{(n-1)}=\mathcal{D} \partial_{s}^{n-1} \xi_{*} & =\frac{1}{2} \sum_{k=1}^{n-1}\left(\begin{array}{l}
n \\
k
\end{array}\right) \partial_{s}^{n-k} z_{*} \partial_{s}^{k} z_{*} \quad(n \geq 2) .
\end{aligned}
$$

The first line of (3.31) follows from

$$
z_{*} \partial_{0} z_{*}=\partial_{0}\left(\xi_{*}-u_{0}\right)=\partial_{0} \xi_{*}-\frac{1}{t}
$$


and

$$
\partial_{0} \xi_{*}=\partial_{s} z_{*}
$$

which follows from (3.18) by differentiating both sides of the equation in $s$. The second line of (3.31) also follows from (3.33). The third line of (3.31) can easily be shown by induction.

It is also useful to note that

$$
\xi_{*}^{(n)}=\partial_{s}^{n} \xi_{*}=\frac{1}{2} \sum_{k=0}^{n}\left(\begin{array}{l}
n \\
k
\end{array}\right) \partial_{s}^{n-k} z_{*} \partial_{s}^{k} z_{*} \quad(n \geq 1),
$$

which immediately follows from (3.8). This relation is important because it enables us to express $\xi_{*}^{(n \geq 1)}$ in terms of

$$
z_{*}^{(n)}:=\partial_{s}^{n} z_{*} \quad(n \geq 1),
$$

and vice versa. For instance, $z_{*}^{(n \geq 1)}$ with small $n$ are expressed in terms of $\xi_{*}^{(n \geq 1)}$ as

$$
\begin{aligned}
& z_{*}^{(1)}=\frac{\xi_{*}^{(1)}}{z_{*}}, \\
& z_{*}^{(2)}=\frac{\xi_{*}^{(2)}}{z_{*}}-\frac{\left(\xi_{*}^{(1)}\right)^{2}}{z_{*}^{3}}, \\
& z_{*}^{(3)}=\frac{\xi_{*}^{(3)}}{z_{*}}-\frac{3 \xi_{*}^{(1)} \xi_{*}^{(2)}}{z_{*}^{3}}+\frac{3\left(\xi_{*}^{(1)}\right)^{3}}{z_{*}^{5}} .
\end{aligned}
$$

Moreover, comparing (3.34) with (3.31) one finds that

$$
\xi_{*}^{(n)}=\mathcal{D} \xi_{*}^{(n-1)}+z_{*} z_{*}^{(n)} \quad(n \geq 1) .
$$

Therefore, by using (3.36) and (3.37) one can express $\mathcal{D} \xi_{*}^{(n \geq 1)}$ as polynomials in $\xi_{*}^{(k \geq 1)}$ and $z_{*}^{-1}$ :

$$
\begin{aligned}
\mathcal{D} \xi_{*}^{(1)} & =\frac{\left(\xi_{*}^{(1)}\right)^{2}}{z_{*}^{2}}, \\
\mathcal{D} \xi_{*}^{(2)} & =\frac{3 \xi_{*}^{(1)} \xi_{*}^{(2)}}{z_{*}^{2}}-\frac{3\left(\xi_{*}^{(1)}\right)^{3}}{z_{*}^{4}}, \\
\mathcal{D} \xi_{*}^{(3)} & =\frac{4 \xi_{*}^{(1)} \xi_{*}^{(3)}}{z_{*}^{2}}+\frac{3\left(\xi_{*}^{(2)}\right)^{2}}{z_{*}^{2}}-\frac{18\left(\xi_{*}^{(1)}\right)^{2} \xi_{*}^{(2)}}{z_{*}^{4}}+\frac{15\left(\xi_{*}^{(1)}\right)^{4}}{z_{*}^{6}} .
\end{aligned}
$$

On the other hand, to evaluate the r.h.s. of (3.29) it is convenient to use

$$
\begin{aligned}
\partial_{0} z_{*} & =\frac{z_{*}^{(1)}}{z_{*}}-\frac{1}{t z_{*}}, \\
\partial_{0} \xi_{*}^{(n)} & =z_{*}^{(n+1)} \quad(n \geq 0) .
\end{aligned}
$$

Again using (3.36) one can express these quantities as polynomials in $t^{-1}, z_{*}^{-1}$ and $\xi_{*}^{(n \geq 1)}$. Hence, by using the low genus results (3.12) and the polynomial structure of $F_{\tilde{g} \geq 2}^{o}$ derived in section 3.2, it is easy to see that all quantities appearing in (3.29) are expressed as polynomials in the variables $t^{-1}, I_{k \geq 2}, z_{*}^{-1},\left(\xi_{*}^{(1)}\right)^{-1}$ and $\xi_{*}^{(n \geq 1)}$. 


\subsection{Higher genus open free energy}

We are now in a position to solve the recursion relation (3.29) and compute the higher genus free energy $F_{\tilde{g}}^{\mathrm{O}}$. To begin with, we verified that $F_{\tilde{g}}^{\mathrm{O}}$ with $\tilde{g}=0,1,2$ given in (3.12) indeed satisfy the recursion relation (3.29) for $\tilde{g}=1,2$. This is easily done by using various identities derived in the last two subsections. Moreover, based on the polynomial structure discussed above, one can perform the integration $\mathcal{D}^{-1}$ completely and determine $F_{\tilde{g}}^{o}$ unambiguously for $\tilde{g} \geq 2$. The algorithm to solve (3.29) and obtain $F_{\tilde{g}}^{o}$ from the data of $\left\{F_{\tilde{g}^{\prime}}^{\text {o }}\right\}_{\tilde{g}^{\prime}<\tilde{g}}$ is as follows:

(i) Compute the r.h.s. of (3.29) using (3.39) and express it as a polynomial in the variables $t^{-1}, I_{k \geq 2}, z_{*}^{-1},\left(\xi_{*}^{(1)}\right)^{-1}$ and $\xi_{*}^{(n \geq 1)}$.

(ii) Let $t^{-m} f\left(I_{k}, z_{*}, \xi_{*}^{(n)}\right)$ denote the highest order part in $t^{-1}$ of the obtained expression. This part can arise only from

$$
\mathcal{D}\left(-\frac{f\left(I_{k}, z_{*}, \xi_{*}^{(n)}\right)}{(m-2) t^{m-2} z_{*} I_{2}}\right)
$$

Therefore subtract this from the obtained expression.

(iii) Repeat the procedure (ii) down to $m=3$. Then all the terms of order $t^{-2}$ automatically disappear and the remaining terms are of order $t^{-1}$ or $t^{0}$. Note also that the expression does not contain any $I_{k}$.

(iv) In the result of (iii), collect all the terms of order $t^{-1}$ and let $t^{-1} \partial_{z_{*}} g\left(z_{*}, \xi_{*}^{(n)}\right)$ denote the sum of them. This part arises from

$$
\mathcal{D} g\left(z_{*}, \xi_{*}^{(n)}\right)
$$

Therefore subtract this from the result of (iii). The remainder turns out to be independent of $t$.

(v) In the obtained expression, let

$$
\frac{h\left(\xi_{*}^{(n \geq 2)}\right)}{z_{*}^{2}\left(\xi_{*}^{(1)}\right)^{m}}
$$

denote the part which is of order $z_{*}^{-2}$ as well as of the lowest order in $\left(\xi_{*}^{(1)}\right)^{-1}$. This part arises from

$$
\mathcal{D}\left(\frac{h\left(\xi_{*}^{(n \geq 2)}\right)}{(m+1)\left(\xi_{*}^{(1)}\right)^{m+1}}\right) .
$$

Therefore subtract this from the obtained expression.

(vi) Repeat the procedure (v) until the resulting expression vanishes. 
(vii) By summing up all the above obtained primitive functions we obtain $F_{\tilde{g}}^{o}$.

Using this algorithm we computed $F_{\tilde{g}}^{\mathrm{o}}$ for $\tilde{g} \leq 15 .{ }^{9}$ We verified that $F_{2}^{\mathrm{o}}$ computed by this algorithm reproduces the result (3.12) of our saddle point calculation. For $\tilde{g}=3$ we obtain

$$
\begin{aligned}
F_{3}^{\mathrm{o}}= & \frac{I_{2}^{2}}{24 t^{4} z_{*}^{2}}+\frac{I_{2}}{8 t^{3} z_{*}^{4}}+\frac{I_{3}}{48 t^{3} z_{*}^{2}}+\frac{5}{16 t^{2} z_{*}^{6}}+\frac{I_{2} \xi_{*}^{(2)}}{48 t^{2} z_{*}^{3} \xi_{*}^{(1)}}-\frac{I_{2} \xi_{*}^{(1)}}{12 t^{2} z_{*}^{5}}+\frac{5 \xi_{*}^{(2)}}{16 t z_{*}^{5} \xi_{*}^{(1)}}-\frac{15 \xi_{*}^{(1)}}{8 t z_{*}^{7}} \\
& -\frac{35 \xi_{*}^{(2)}}{16 z_{*}^{6}}+\frac{35\left(\xi_{*}^{(1)}\right)^{2}}{8 z_{*}^{8}}-\frac{\xi_{*}^{(4)}}{16 z_{*}^{2}\left(\xi_{*}^{(1)}\right)^{2}}+\frac{5 \xi_{*}^{(3)}}{16 z_{*}^{4} \xi_{*}^{(1)}}-\frac{3\left(\xi_{*}^{(2)}\right)^{2}}{16 z_{*}^{4}\left(\xi_{*}^{(1)}\right)^{2}}-\frac{\left(\xi_{*}^{(2)}\right)^{3}}{4 z_{*}^{2}\left(\xi_{*}^{(1)}\right)^{4}} \\
& +\frac{7 \xi_{*}^{(3)} \xi_{*}^{(2)}}{24 z_{*}^{2}\left(\xi_{*}^{(1)}\right)^{3}}+\frac{\xi_{*}^{(5)}}{48\left(\xi_{*}^{(1)}\right)^{3}}-\frac{\left(\xi_{*}^{(3)}\right)^{2}}{8\left(\xi_{*}^{(1)}\right)^{4}}-\frac{\xi_{*}^{(4)} \xi_{*}^{(2)}}{6\left(\xi_{*}^{(1)}\right)^{4}}+\frac{3 \xi_{*}^{(3)}\left(\xi_{*}^{(2)}\right)^{2}}{4\left(\xi_{*}^{(1)}\right)^{5}}-\frac{\left(\xi_{*}^{(2)}\right)^{4}}{2\left(\xi_{*}^{(1)}\right)^{6}}
\end{aligned}
$$

\section{Conclusions and outlook}

In this paper we have studied the small $g_{\mathrm{s}}$ expansion (1.3) of the open free energy $F^{\circ}(s)$ of topological gravity. We have obtained the explicit form (3.23) of the genus zero part $F_{0}^{o}$ of the free energy. We have then argued that the higher order corrections $F_{\tilde{g}}^{o}$ can be computed systematically by solving Buryak's equation recursively. We have demonstrated this computation explicitly for the first few orders. We have also elucidated the polynomial structure of $F_{\tilde{g} \geq 2}^{\mathrm{o}}$. We emphasize that our result of $F_{\tilde{g}}^{o}$ holds for arbitrary value of the couplings $\left\{t_{n}\right\}$. We have shown that $F_{\tilde{g}}^{o}$ is written as a combination of genus-zero quantities only, which can be thought of as an open analog of the constitutive relation for closed topological gravity [26, 27].

The existence of the polynomial structure established in this paper is important in several respects. From a practical viewpoint, it is this structure that enables us to determine the free energy merely by solving a single, simple differential equation rather than an infinite number of KdV flow equations or Virasoro constraints. From a philosophical perspective, our study would serve as a simple example of Gromov-Witten theories in which the polynomial structure of the closed sector naturally extends to the open sector. It could uncover the existence of similar polynomial structures in a broader class of open GromovWitten theories. Since our derivation of the polynomial structure is simply based on the relation (1.2) or (3.5) between the open free energy and the BA function, it is likely to be generalized at least to some cases such as the theory of open $r$-spin intersection numbers [34] for which a similar relation is known [35]. We stress that the polynomial structure is of the type known as the constitutive relation, i.e. all the generators are genus zero quantities. All these may suggest the possibility of an open analog of Givental's formalism [36-38], namely a general framework of determining higher genus open Gromov-Witten invariants from genus zero ones.

There are several interesting open questions. In [39] a refinement of the open intersection numbers is presented, where how boundary markings are partitioned between the boundaries is taken into account. It is conjectured [39] that the refined open free energy,

\footnotetext{
${ }^{9}$ The data of $F_{\tilde{g}}^{o}$ are available upon request to the authors.
} 
or the associated partition function $\tau_{N}^{\text {o,ext }}$, is written in terms of the Kontsevich-Penner matrix integral, generalizing the result established in the $N=1$ unrefined case [20,21]. A similar matrix integral that is deformed by an additional parameter $N$ is considered for the Brezin-Gross-Witten model [40, 41]. Its genus expansion is computed both in the finite $N$ regime and in the 't Hooft regime: $N \gg 1, g_{\mathrm{s}} \ll 1$ with $g_{\mathrm{s}} N$ fixed [31, 41]. In particular, the genus expansion in the latter regime can be computed by simply solving the $\mathrm{KdV}$ equation [31]. It is interesting to see if the refined open free energy can similarly be computed in the 't Hooft regime by the techniques developed in this paper.

In general, the small $g_{\mathrm{s}}$ expansion of $F^{\mathrm{o}}$ in (1.3) is an asymptotic series and we expect that $F^{\mathrm{o}}$ receives non-perturbative corrections in $g_{\mathrm{s}}$. Such corrections are physically interpreted as the effect of the so-called ZZ-branes [42]. It would be interesting to find the general structure of the effect of ZZ-branes for the arbitrary background $\left\{t_{n}\right\}$. It is known that [43] some of the background $\left\{t_{n}\right\}$ exhibits a non-perturbative instability and it does not lead to a well-defined theory. It would be interesting to find the map of the "swampland" in the space of all two-dimensional topological gravities $\left\{t_{n}\right\}$. In particular, it is argued that the JT gravity matrix model suffers from such a non-perturbative instability [11]. It is important to see if JT gravity is non-perturbatively well-defined or not.

\section{Acknowledgments}

This work was supported in part by JSPS KAKENHI Grant Nos. 19K03845 and 19K03856, and JSPS Japan-Russia Research Cooperative Program.

\section{A Derivation of open $\mathrm{KdV}$ equations}

In this section we will derive the open $\mathrm{KdV}$ equations (2.28) from the $\mathrm{KdV}$ flow equations (2.35).

Let $n$ be a positive integer. Since

$$
Q^{n+\frac{1}{2}}=Q \cdot Q^{n-\frac{1}{2}}=Q\left(Q_{+}^{n-\frac{1}{2}}+Q_{-}^{n-\frac{1}{2}}\right),
$$

we have

$$
Q_{+}^{n+\frac{1}{2}}=\left(Q \cdot Q_{+}^{n-\frac{1}{2}}\right)_{+}+\left(Q \cdot Q_{-}^{n-\frac{1}{2}}\right)_{+}=Q \cdot Q_{+}^{n-\frac{1}{2}}+\left(Q \cdot Q_{-}^{n-\frac{1}{2}}\right)_{+} .
$$

Therefore (2.35) is rewritten as

$$
\frac{2 n+1}{2} \partial_{n} \widehat{\psi}=Q \frac{(2 Q)_{+}^{n-\frac{1}{2}}}{(2 n-1) ! ! g_{\mathrm{s}}} \widehat{\psi}+\left(Q \frac{(2 Q)_{-}^{n-\frac{1}{2}}}{(2 n-1) ! ! g_{\mathrm{s}}}\right)_{+} \widehat{\psi}
$$

Using (2.35) again one finds that the first term on the r.h.s. of (A.3) gives $Q \partial_{n-1} \widehat{\psi}$. On the other hand, it is known that (see e.g. [28]) $Q_{-}^{n-\frac{1}{2}}$ has the structure

$$
Q_{-}^{n-\frac{1}{2}}=\frac{(2 n-1) ! !}{2^{n+\frac{1}{2}} g_{\mathrm{s}}}\left\{\mathcal{R}_{n}, \partial_{0}^{-1}\right\}+\mathcal{O}\left(\partial_{0}^{-3}\right)
$$


from which (2.13) follows. By using this, the second term on the r.h.s. of (A.3) becomes

$$
\begin{aligned}
\left(Q \frac{(2 Q)_{-}^{n-\frac{1}{2}}}{(2 n-1) ! ! g_{\mathrm{s}}}\right)_{+} \widehat{\psi} & =\frac{1}{4}\left(\partial_{0}^{2}\left\{\mathcal{R}_{n}, \partial_{0}^{-1}\right\}\right)_{+} \widehat{\psi} \\
& =\frac{1}{4}\left(\left(\partial_{0}^{2} \mathcal{R}_{n}\right) \partial_{0}^{-1}+\left(\partial_{0} \mathcal{R}_{n}\right)+2 \partial_{0} \mathcal{R}_{n}\right)_{+} \widehat{\psi} \\
& =\frac{1}{4}\left(\left(\partial_{0} \mathcal{R}_{n}\right) \widehat{\psi}+2 \partial_{0}\left(\mathcal{R}_{n} \widehat{\psi}\right)\right) \\
& =\frac{3}{4}\left(\partial_{0} \mathcal{R}_{n}\right) \widehat{\psi}+\frac{1}{2} \mathcal{R}_{n} \partial_{0} \widehat{\psi}
\end{aligned}
$$

Hence (A.3) becomes

$$
\begin{aligned}
\frac{2 n+1}{2} \partial_{n} \widehat{\psi} & =Q \partial_{n-1} \widehat{\psi}+\frac{3}{4}\left(\partial_{0} \mathcal{R}_{n}\right) \widehat{\psi}+\frac{1}{2} \mathcal{R}_{n} \partial_{0} \widehat{\psi} \\
& =\left(\frac{g_{\mathrm{s}}^{2}}{2} \partial_{0}^{2}+u\right) \partial_{n-1} \widehat{\psi}+\frac{3}{4}\left(\partial_{n-1} u\right) \widehat{\psi}+\frac{g_{\mathrm{s}}^{2}}{2}\left(\partial_{0} \partial_{n-1} F^{\mathrm{c}}\right) \partial_{0} \widehat{\psi} \\
& =\partial_{n-1}\left(\frac{g_{\mathrm{s}}^{2}}{2} \partial_{0}^{2}+u\right) \widehat{\psi}-\frac{1}{4}\left(\partial_{n-1} u\right) \widehat{\psi}+\frac{g_{\mathrm{s}}^{2}}{2}\left(\partial_{0} \partial_{n-1} F^{\mathrm{c}}\right) \partial_{0} \widehat{\psi} \\
& =\partial_{n-1} Q \widehat{\psi}+\frac{g_{\mathrm{s}}^{2}}{2}\left(\partial_{0} \partial_{n-1} F^{\mathrm{c}}\right) \partial_{0} \widehat{\psi}-\frac{1}{4}\left(\partial_{n-1} u\right) \widehat{\psi}
\end{aligned}
$$

In the second equality we have used (2.5) and (2.9). Substituting (2.32) we have

$$
\frac{2 n+1}{2} \partial_{n} \widehat{\psi}=g_{\mathrm{s}} \partial_{s} \partial_{n-1} \widehat{\psi}+\frac{g_{\mathrm{s}}^{2}}{2}\left(\partial_{0} \partial_{n-1} F^{\mathrm{c}}\right) \partial_{0} \widehat{\psi}-\frac{g_{\mathrm{s}}^{2}}{4}\left(\partial_{0}^{2} \partial_{n-1} F^{\mathrm{c}}\right) \widehat{\psi}
$$

Under the identification (2.33) one sees that this is equivalent to the open KdV equations (2.28).

\section{B Derivation of $z_{*}(s)$}

In this section we will derive (3.9) from (3.7).

Suppose that $w$ is expressed as a function of $z$ given by the formal power series

$$
w=f(z)=\sum_{n=1}^{\infty} f_{n} \frac{z^{n}}{n !}
$$

with $f_{1} \neq 0$. According to the Lagrange inversion theorem, the inverse function is given by

$$
z=g(w)=\sum_{n=1}^{\infty} g_{n} \frac{w^{n}}{n !}
$$

with

$$
g_{1}=\frac{1}{f_{1}}, \quad g_{n}=\frac{1}{f_{1}^{n}} \sum_{k=1}^{n-1}(-1)^{k}(n+k-1) ! \sum_{\left\{j_{\ell}\right\}} \prod_{\ell=1}^{n-k} \frac{1}{j_{\ell}}\left(\frac{f_{\ell+1}}{(\ell+1) ! f_{1}}\right)^{j_{\ell}} \quad(n \geq 2),
$$


where the second sum is taken over all sequences $j_{1}, j_{2}, \ldots, j_{n-k}$ of non-negative integers such that

$$
\begin{aligned}
j_{1}+j_{2}+\cdots+j_{n-k} & =k, \\
j_{1}+2 j_{2}+\cdots+(n-k) j_{n-k} & =n-1 .
\end{aligned}
$$

In the present case we have

$$
\begin{array}{ll}
w=s, & z=z_{*}, \\
f_{1}=t, & \frac{f_{n}}{n !}=\left\{\begin{array}{cc}
-\frac{1}{n ! !} I_{\frac{n+1}{2}} & n=3,5,7, \ldots, \\
0 & n=2,4,6, \ldots
\end{array}\right.
\end{array}
$$

Since $f_{\ell+1}$ with odd $\ell$ are absent, the conditions (B.4) reduce to

$$
\begin{aligned}
& j_{2}+j_{4}+\cdots+j_{2\left\lfloor\frac{n-k}{2}\right\rfloor}=k, \\
& 2 j_{2}+4 j_{4}+\cdots+2\left\lfloor\frac{n-k}{2}\right\rfloor j_{2\left\lfloor\frac{n-k}{2}\right\rfloor}=n-1 .
\end{aligned}
$$

It is clear that the second condition is satisfied only if $n$ is odd. This means that all $g_{n}$ with even $n$ vanish. For odd $n(\geq 3)$ we have

$$
\begin{aligned}
g_{n} & =\frac{1}{t^{n}} \sum_{k=1}^{n-1}(-1)^{k}(n+k-1) ! \sum_{\left\{j_{2 a}\right\}} \prod_{a=1}^{\left.\frac{n-k}{2}\right\rfloor} \frac{1}{j_{2 a} !}\left(-\frac{I_{a+1}}{(2 a+1) ! ! t}\right)^{j_{2 a}} \\
& =\sum_{k=1}^{n-1} \frac{(n+k-1) !}{t^{n+k}} \sum_{\left\{j_{2 a}\right\}} \prod_{a=1}^{\left\lfloor\frac{n-k}{2}\right\rfloor} \frac{1}{j_{2 a} !}\left(\frac{I_{a+1}}{(2 a+1) ! !}\right)^{j_{2 a}} .
\end{aligned}
$$

Therefore

$$
\begin{aligned}
& z_{*}= \frac{s}{t}+\sum_{m=1}^{\infty} \frac{s^{2 m+1}}{(2 m+1) !} g_{2 m+1} \\
&= \frac{s}{t}+\sum_{m=1}^{\infty} \frac{s^{2 m+1}}{(2 m+1) !} \sum_{k=1}^{2 m} \frac{(2 m+k) !}{t^{2 m+k+1}} \sum_{\left\{j_{2 a}\right\}} \prod_{a=1}^{\left\lfloor\frac{2 m-k+1}{2}\right\rfloor} \frac{1}{j_{2 a} !}\left(\frac{I_{a+1}}{(2 a+1) ! !}\right)^{j_{2 a}} \\
&= \frac{s}{t}+\sum_{j_{2 a} \geq 0} \frac{(2 m+k) !}{(2 m+1) !} \frac{s^{2 m+1}}{t^{2 m+k+1}} \prod_{a=1}^{\infty} \frac{1}{j_{2 a} !}\left(\frac{I_{a+1}}{(2 a+1) ! !}\right)^{j_{2 a}} \\
&= \sum_{\sum_{a} j_{2 a}=k \geq 1} \sum_{j_{2 a} \geq 0} \frac{(2 m+k) !}{(2 m+1) !} \frac{s^{2 m+1}}{t^{2 m+k+1}} \prod_{a=1}^{\infty} \frac{1}{j_{2 a} !}\left(\frac{I_{a+1}}{(2 a+1) ! !}\right)^{j_{2 a}} . \\
& \sum_{a} j_{2 a}=k \\
& \sum_{a} a j_{2 a}=m
\end{aligned}
$$

By rewriting $j_{2 a}$ as $j_{a}$ this gives (3.9). 
Open Access. This article is distributed under the terms of the Creative Commons Attribution License (CC-BY 4.0), which permits any use, distribution and reproduction in any medium, provided the original author(s) and source are credited.

\section{References}

[1] D.J. Gross and A.A. Migdal, Nonperturbative Two-Dimensional Quantum Gravity, Phys. Rev. Lett. 64 (1990) 127 [INSPIRE].

[2] D.J. Gross and A.A. Migdal, A Nonperturbative Treatment of Two-dimensional Quantum Gravity, Nucl. Phys. B 340 (1990) 333 [INSPIRE].

[3] M.R. Douglas and S.H. Shenker, Strings in Less Than One-Dimension, Nucl. Phys. B 335 (1990) 635 [INSPIRE].

[4] E. Brézin and V.A. Kazakov, Exactly Solvable Field Theories of Closed Strings, Phys. Lett. B 236 (1990) 144 [INSPIRE].

[5] P.H. Ginsparg and G.W. Moore, Lectures on 2-D gravity and 2-D string theory, in Theoretical Advanced Study Institute (TASI 92): From Black Holes and Strings to Particles, (1993) [hep-th/9304011] [INSPIRE].

[6] E. Witten, Two-dimensional gravity and intersection theory on moduli space, Surveys Diff. Geom. 1 (1991) 243.

[7] M. Kontsevich, Intersection theory on the moduli space of curves and the matrix Airy function, Commun. Math. Phys. 147 (1992) 1 [INSPIRE].

[8] M.R. Douglas, Strings in Less Than One-dimension and the Generalized $K^{-} D^{-} V$ Hierarchies, Phys. Lett. B 238 (1990) 176 [INSPIRE].

[9] M. Fukuma, H. Kawai and R. Nakayama, Continuum Schwinger-dyson Equations and Universal Structures in Two-dimensional Quantum Gravity, Int. J. Mod. Phys. A 6 (1991) 1385 [INSPIRE].

[10] R. Dijkgraaf, H.L. Verlinde and E.P. Verlinde, Loop equations and Virasoro constraints in nonperturbative 2-D quantum gravity, Nucl. Phys. B 348 (1991) 435 [INSPIRE].

[11] P. Saad, S.H. Shenker and D. Stanford, JT gravity as a matrix integral, arXiv:1903.11115 [INSPIRE].

[12] M. Mulase and B. Safnuk, Mirzakhani's recursion relations, Virasoro constraints and the KdV hierarchy, math/0601194 [INSPIRE].

[13] R. Dijkgraaf and E. Witten, Developments in Topological Gravity, Int. J. Mod. Phys. A 33 (2018) 1830029 [arXiv: 1804.03275] [inSPIRE].

[14] K. Okuyama and K. Sakai, JT gravity, KdV equations and macroscopic loop operators, JHEP 01 (2020) 156 [arXiv:1911.01659] [INSPIRE].

[15] R. Pandharipande, J.P. Solomon and R.J. Tessler, Intersection theory on moduli of disks, open KdV and Virasoro, arXiv:1409.2191 [INSPIRE].

[16] A. Buryak, Open intersection numbers and the wave function of the KdV hierarchy, Moscow Math. J. 16 (2016) 27 [arXiv:1409.7957] [INSPIRE]. 
[17] A. Buryak, Equivalence of the open KdV and the open Virasoro equations for the moduli space of Riemann surfaces with boundary, Lett. Math. Phys. 105 (2015) 1427 [arXiv: 1409.3888] [INSPIRE].

[18] A. Buryak and R.J. Tessler, Matrix Models and A Proof of the Open Analog of Witten's Conjecture, Commun. Math. Phys. 353 (2017) 1299 [arXiv:1501.07888] [INSPIRE].

[19] R.J. Tessler, The combinatorial formula for open gravitational descendents, arXiv: 1507.04951 [INSPIRE].

[20] A. Alexandrov, Open intersection numbers, matrix models and MKP hierarchy, JHEP 03 (2015) 042 [arXiv: 1410.1820] [INSPIRE].

[21] A. Alexandrov, Open intersection numbers, Kontsevich-Penner model and cut-and-join operators, JHEP 08 (2015) 028 [arXiv:1412.3772] [INSPIRE].

[22] K. Okuyama and K. Sakai, Multi-boundary correlators in JT gravity, JHEP 08 (2020) 126 [arXiv:2004.07555] [INSPIRE].

[23] V. Fateev, A.B. Zamolodchikov and A.B. Zamolodchikov, Boundary Liouville field theory. 1. Boundary state and boundary two point function, hep-th/0001012 [INSPIRE].

[24] J. Teschner, Remarks on Liouville theory with boundary, PoS tmr2000 (2000) 041 [hep-th/0009138] [INSPIRE].

[25] J.M. Maldacena, G.W. Moore, N. Seiberg and D. Shih, Exact vs. semiclassical target space of the minimal string, JHEP 10 (2004) 020 [hep-th/0408039] [INSPIRE].

[26] C. Itzykson and J.B. Zuber, Combinatorics of the modular group. 2. The Kontsevich integrals, Int. J. Mod. Phys. A 7 (1992) 5661 [hep-th/9201001] [INSPIRE].

[27] T. Eguchi, Y. Yamada and S.-K. Yang, On the genus expansion in the topological string theory, Rev. Math. Phys. 7 (1995) 279 [hep-th/9405106] [InSPIRE].

[28] P. Di Francesco, P.H. Ginsparg and J. Zinn-Justin, 2-D Gravity and random matrices, Phys. Rept. 254 (1995) 1 [hep-th/9306153] [INSPIRE].

[29] Q. Zhang and J. Zhou, On Itzykson-Zuber Ansatz, JHEP 09 (2019) 075 [arXiv:1904.09071] [INSPIRE].

[30] P. Zograf, On the large genus asymptotics of Weil-Petersson volumes, arXiv:0812.0544 [INSPIRE].

[31] K. Okuyama and K. Sakai, JT supergravity and Brezin-Gross-Witten tau-function, JHEP 10 (2020) 160 [arXiv: 2007.09606] [InSPIRE].

[32] R. Dijkgraaf and E. Witten, Mean Field Theory, Topological Field Theory, and Multimatrix Models, Nucl. Phys. B 342 (1990) 486 [InSPIRE].

[33] J. Zhou, On Topological 1D Gravity. I, arXiv:1412.1604 [INSPIRE].

[34] A. Buryak, E. Clader and R.J. Tessler, Open r-spin theory II: The analogue of Witten's conjecture for r-spin disks, arXiv:1809.02536.

[35] M. Bertola and D. Yang, The partition function of the extended $r$-reduced Kadomtsev-Petviashvili hierarchy, J. Phys. A 48 (2015) 195205 [arXiv:1411.5717] [INSPIRE].

[36] A. Givental, Semisimple Frobenius structures at higher genus, Int. Math. Res. Not. 2001 (2001) 1265 [math/0008067] [INSPIRE]. 
[37] A.B. Givental, Gromov-Witten invariants and quantization of quadratic hamiltonians, Moscow Math. J. 1 (2001) 551 [math/0108100] [INSPIRE].

[38] T. Coates and A. Givental, Quantum Riemann-Roch, Lefschetz and Serre, Ann. Math. 165 (2007) 15 [math/0110142] [INSPIRE].

[39] A. Alexandrov, A. Buryak and R.J. Tessler, Refined open intersection numbers and the Kontsevich-Penner matrix model, JHEP 03 (2017) 123 [arXiv: 1702.02319] [INSPIRE].

[40] A. Mironov, A. Morozov and G.W. Semenoff, Unitary matrix integrals in the framework of generalized Kontsevich model. 1. Brezin-Gross-Witten model, Int. J. Mod. Phys. A 11 (1996) 5031 [hep-th/9404005] [INSPIRE].

[41] A. Alexandrov, Cut-and-join description of generalized Brezin-Gross-Witten model, Adv. Theor. Math. Phys. 22 (2018) 1347 [arXiv:1608.01627] [InSPIRE].

[42] A.B. Zamolodchikov and A.B. Zamolodchikov, Liouville field theory on a pseudosphere, hep-th/0101152 [INSPIRE].

[43] N. Seiberg and D. Shih, Branes, rings and matrix models in minimal (super)string theory, JHEP 02 (2004) 021 [hep-th/0312170] [INSPIRE]. 